NOUVELLE

\section{Par ici la sortie! \\ Le SARS-CoV-2 utilise \\ les lysosomes pour sortir \\ de la cellule infectée}

Riddhi Atul Jani ${ }^{\star}$, Laura Salavessa ${ }^{*}$, Cédric Delevoye
$>$ Depuis les premiers signalements de patients atteints de pneumonie virale en République populaire de Chine à la fin de l'année 2019, la pandémie actuelle de COVID-19 (coronavirus disease 2019) a entraîné une crise sanitaire mondiale. Le responsable est un nouveau $\beta$-coronavirus, isolé au début de l'année 2020 [1]

$(\rightarrow)$ Voir la Synthèse de F. Iseni et J.N. Tournier, $m / s n^{\circ} 8-9$, août$(\rightarrow)$, à l'origine septembre 2020, page 797

d'un syndrome respiratoire aigu sévère (severe acute respiratory syndrome ou SARS) : le SARS-CoV-2 (SARS coronavirus 2). C'est un virus enveloppé à ARN simple brin positif, reconnaissable en microscopie électronique grâce aux projections de glycoprotéine $S$ (spike) qui ornent sa surface, ressemblant à une couronne (corona). Plus généralement, les virus de la famille Coronaviridae représentent un enjeu sanitaire majeur car ils sont souvent responsables de maladies respiratoires ou intestinales hautement transmissibles.

En tant que parasite intracellulaire obligatoire, le virus détourne la machinerie d'une cellule hôte pour se reproduire. Cette cellule hôte contient différents compartiments membranaires, ou organites, qui échangent divers composants en utilisant les voies du trafic intracellulaire. Parmi elles, la voie de sécrétion achemine des protéines néo-synthétisées à travers le réticulum endoplasmique, l'appareil de Golgi, et les vésicules d'exocytose, lesquelles sont transportées à la périphérie cellulaire et fusionnent avec la membrane plasmique pour libérer leur contenu dans l'espace extracellulaire. La voie d'endocytose permet également l'absorption de molécules solubles et membranaires, qui sont triées dans les endosomes avant de retourner à la surface cellulaire ou d'être dégradées dans les lysosomes, des organites à activité protéolytique et au $\mathrm{pH}$ acide. Lors du cycle infectieux, un virus peut exploiter ces organites et ces voies du trafic intracellulaire comme véhicules et chemins de sortie de la cellule hôte afin de transmettre de nouveaux virions aux cellules voisines. De nombreux virus enveloppés sortent de la cellule en empruntant la voie de sécrétion [2], mais les résultats d'une étude récente suggèrent que les coronavirus et le SARS-CoV-2 utilisent le lysosome comme voie de sortie alternative [3].

La clé d'entrée cellulaire du SARS-CoV-2 est sa protéine spike, qui facilite son internalisation en se liant au récepteur ACE2 (enzyme de conversion de l'angiotensine 2) (Figure 1) [4]. Une fois internalisé, le virus réside dans une vésicule d'endocytose, qu'il quitte rapidement pour libérer, dans le cytosol de la cellule hôte, son matériel génétique. Ce génome viral est traduit en protéines permettant la production d'ARN génomiques viraux, lesquels contribueront à la formation de nouveaux virions par l'exploitation de membranes dérivées du réticulum endoplasmique et de l'appareil de Golgi (Figure 1). C'est là que débute l'étude de Ghosh et al. [3], qui explore comment les virions d'un $\beta$-coronavirus murin, le virus de l'hépatite de souris (mouse hepatitis virus, MHV), s'échappent de la cellule hôte. En étudiant la voie de sécrétion,
CNRS UMR 144, Structure et compartiments membranaires, Institut Curie, université de recherche $P S L$,

12 rue Lhomond, 75005 Paris, France.

*Ces auteurs ont contribué à cet article à parts égales cedric.delevoye@curie.fr

ces auteurs ont constaté que sa perturbation par la bréfeldine $A$, qui interrompt le transport entre le réticulum endoplasmique et l'appareil de Golgi, n'affecte pas la sortie des virions. Par microscopie à fluorescence de cellules infectées par le virus, ces auteurs ont également montré que certaines protéines virales sont partiellement co-localisées avec des composants couramment associés avec les lysosomes. Bien que cela puisse refléter la capacité de la cellule hôte à «digérer » le virus, des virions d'apparence intacte sont identifiés, par microscopie électronique, à l'intérieur de compartiments ressemblant morphologiquement à des lysosomes. Cette observation suggérait que les particules virales pourraient résister à la dégradation dans ces organites. En utilisant des sondes fluorescentes ciblant les organites au $\mathrm{pH}$ acide ou à activité protéolytique, les auteurs ont découvert une alcalinisation des lysosomes des cellules infectées, à laquelle est associée une moindre activité de dégradation protéolytique. Ces lysosomes sont donc dysfonctionnels, mais peuvent-ils jouer le rôle de véhicules guidant les virions vers la sortie cellulaire? Par des approches d'inactivation de gènes, les auteurs ont identifié la petite GTPase Arl8b, connue pour faciliter le transport des lysosomes vers la périphérie [5], comme un composant de la cellule hôte nécessaire à la sortie des virions. Les lysosomes dysfonctionnels contenant des virions utiliseraient cette GTPase pour s'approcher de la membrane plasmique et faciliter l'exocytose des particules virales. 


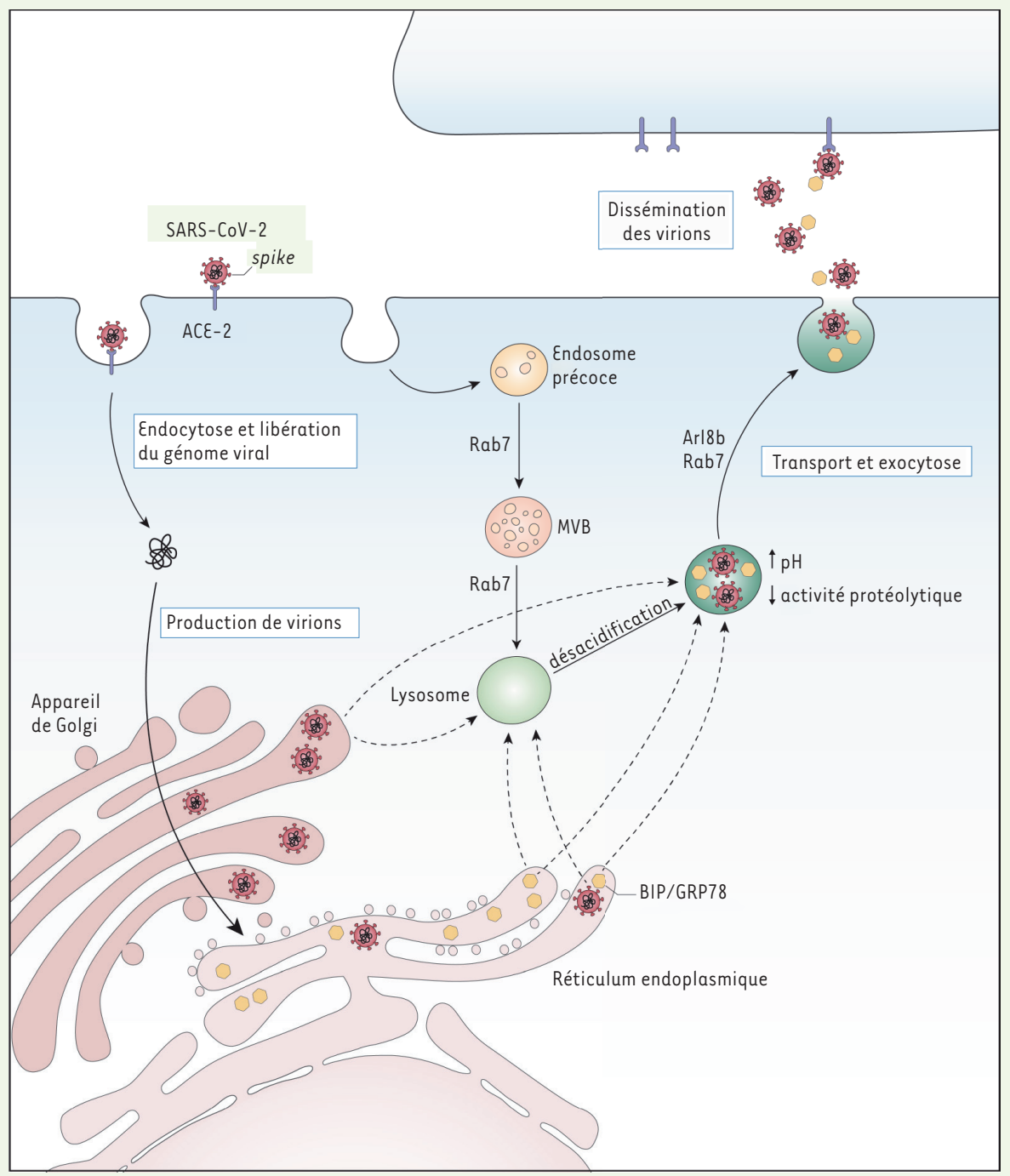

Figure 1. Le SARS-CoV-2 utilise un lysosome modifié pour sortir de la cellule. Le SARS-CoV-2 infecte une cellule humaine en y entrant par endocytose, grâce à la liaison de sa protéine spike au récepteur ACE2. Une fois le virus internalisé, la libération de son matériel génétique dans le cytosol servira à la production de nouveaux virions par l'exploitation des membranes cellulaires du réticulum endoplasmique et de l'appareil de Golgi. Le long de la voie d'endocytose, composée d'endosomes précoces, de corps multi-vésiculaires (MVB) et de lysosomes, le SARS-CoV-2 exploiterait les lysosomes pour sa sortie. L'infection virale augmente le $\mathrm{pH}$ luminal des lysosomes et diminue leur activité protéolytique. On ignore comment les particules virales rejoignent les lysosomes, et si elles les modifient «de l'intérieur» ou « à distance » (flèches en trait pointillé). Ces lysosomes chargés de particules virales utilisent des machineries cellulaires (Arl8 et Rab7) pour faciliter leur transport à la membrane plasmique et l'exocytose des virions. La libération des virions nouvellement

formés est concomitante à la sécrétion de la protéine chaperon GRP78/BIP, qui aiderait au repliement des protéines virales telles que spike, afin d'optimiser sa reconnaissance par le récepteur ACE2 pour infecter d'autres cellules. Le mécanisme d'enrichissement des lysosomes en GRP78/BIP, une protéine du réticulum endoplasmique, reste à élucider.

Étonnamment, ces virions sont libérés concomitamment à la protéine chaperon GRP78/BIP, qui ajuste, dans la lumière du réticulum endoplasmique, le repliement de protéines empruntant notamment la voie de sécrétion [6]. Cette libération conjointe des virions et de GRP78/BIP indiquerait qu'il existe une connexion étroite, bien que mal comprise, entre la voie de sécrétion et les lysosomes. D'un point de vue fonctionnel, les auteurs proposent que le dérèglement des lysosomes des cellules infectées par le virus altère la présentation des antigènes viraux aux cellules du système immunitaire, ce qui nuirait à la défense de l'organisme contre l'infection.

Ces travaux mettent en lumière des mécanismes cellulaires et moléculaires employés par un $\beta$-coronavirus, tel que le SARS-CoV-2, pour se propager. En exploitant les lysosomes de la cellule hôte, ce virus aurait acquis l'avantage d'un mécanisme d'échappement, pas encore décrit pour les virus enveloppés (Figure 1). Au sein des Coronaviridae, l'usurpation du lysosome par le virus contribue-t-elle à «l'efficacité »de son cycle infectieux? D'autres coronavirus sont sécrétés simultanément avec GRP78/BIP, ce qui optimiserait le repliement de la protéine spike et sa liaison à $A C E 2$, rendant ainsi le virus plus infectieux $[7,8]$. Ainsi, le cycle infectieux du SARS-CoV-2 comporterait des caractéristiques cellulaires communes aux Coronaviridae, mais aussi d'autres, nouvellement acquises, qui, en ciblant le lysosome, interféreraient avec la réponse immunitaire de l'hôte et contribueraient à la pathogenèse de la COVID-19. 
Cette étude présente cependant des limites qu'il convient d'examiner. L'une d'elles est l'emploi du MHV plutôt que du SARS-CoV-2 lui-même (pour des raisons de biosécurité). Bien que génétiquement proches, MHV et SARS-CoV-2 appartiennent à des sous-genres différents de $\beta$-coronavirus (Embecovirus et Sarbecovirus, respectivement), qui ont pu diverger et acquérir de nouvelles propriétés. Cependant, les auteurs ont validé certaines de leurs observations, comme la présence de composants viraux dans les lysosomes, sur des cellules infectées par le SARS-CoV-2. L'identité précise de l'organite hôte du MHV ou du SARS-CoV-2 reste cependant à définir. $\varepsilon n$ effet, et bien qu'appartenant à la lignée des lysosomes, les compartiments contenant les virions pourrait être des corps multi-vésiculaires, des endo-lysosomes, ou des organites apparentés aux lysosomes [9]. Les auteurs excluent les corps multi-vésiculaires, car la perturbation expérimentale de leur biogenèse, transport ou exocytose n'a pas eu d'impact sur la sortie des virions. Cependant, les traitements utilisés dans l'étude pourraient n'avoir affecté qu'une souspopulation de corps multi-vésiculaires. Les auteurs ont cherché à assigner une identité moléculaire plus précise à ce compartiment viral en identifiant la présence des marqueurs LAMPI ou Rab7, mais ces marqueurs sont aussi présents dans de nombreux compartiments de la lignée des lysosomes sus-mentionnés [10]. Toutefois, il faut noter qu'un traitement par un antagoniste de Rab7 altérant la formation et le transport des lysosomes [10] limite la sortie du virus. Ce résultat, quoique prometteur, devra être confirmé car cet antagoniste est un inhibiteur à large spectre des protéines de la famille Ras, incluant Rab7 [11]. Néanmoins, de tels inhibiteurs pourraient constituer une nouvelle classe d'agents thérapeutiques capables d'entraver la propagation virale.

Si l'identité des organites dans lesquels résident MHV ou SARS-CoV-2 reste imprécise, le mécanisme permet- tant à la protéine chaperon GRP78/ BIP de quitter la lumière du réticulum endoplasmique pour rejoindre celle des lysosomes chargés de virions est encore plus énigmatique. Compte tenu des nombreux contacts membranaires entre le réticulum endoplasmique et les lysosomes [12], s'agit-il d'un échange non vésiculaire de molécules, ou, au contraire, d'un transport membranaire non conventionnel, entre ces deux compartiments cellulaires (Figure 1)? Par ailleurs, les virions issus des membranes du réticulum endoplasmique pourraientils être «ingérés » par les lysosomes? Si c'est le cas, ces lysosomes sont-ils pré-alcalinisés, ou leur $\mathrm{pH}$ acide est-il neutralisé après l'absorption du virus? Enfin, quel est le mécanisme de «l'attaque » des lysosomes par le virus? (Figure 1). Toutes ces questions encore sans réponse ouvrent un large champ d'investigations nécessaires à la clarification du parcours intracellulaire $d u$ SARS-CoV-2.

L'utilisation des lysosomes comme porte de sortie du SARS-CoV-2 représente une nouveauté dans le domaine de l'infection par les virus enveloppés, mais aussi une opportunité pour mieux appréhender l'origine des symptômes cliniques de la COVID-19 et pour identifier des médicaments afin de la combattre. Le dérèglement des lysosomes par alcalinisation, en tant que processus infectieux, tend à éliminer l'usage de bases faibles et d'agents «lysosomotropes», comme la chloroquine et ses dérivés, qui pourraient au contraire favoriser la propagation virale en facilitant la désacidification de ces organites. La cosécrétion de GRP78/BIP avec les virions de SARS-CoV-2 rappelle celle observée pour d'autres virus (Zika, Ebola, virus de la dengue), dont l'entrée cellulaire ou la production dans la cellule hôte est affectée par des médicaments ciblant cette protéine chaperon, suggérant la possibilité d'une réutilisation de tels médicaments contre la COVID-19. Les auteurs de cette étude sur le MHV proposent également que la présentation des antigènes viraux et l'activation des lymphocytes $T$ soient réduites suite au dérèglement des lysosomes, lui-même nécessaire au processus de sécrétion des virions par les cellules infectées. Une telle connexion entre le cycle intracellulaire du virus et l'activation du système de défense immunitaire ouvre des possibilités de mieux comprendre les complications observées chez les patients développant des formes sévères de la COVID-19. Ces formes pouvant résulter d'une réponse immunitaire affaiblie ou dérégulée, cette étude pourrait contribuer au développement de nouveaux traitements par des molécules immunomodulatrices.

Cette étude illustre, s'il était besoin, l'importance de décrypter le cycle cellulaire du SARS-CoV-2 pour progresser dans notre compréhension de la physiopathologie de la COVID-19, identifier de nouvelles cibles thérapeutiques, et éventuellement repositionner des médicaments existants pour lutter contre cette maladie. Les biologistes du monde entier cherchent à comprendre comment l'infection par le SARS-CoV-2 module le fonctionnement de la cellule hôte, en caractérisant par exemple comment les protéines virales exploitent individuellement les composants cellulaires [13] ou les organites [14] afin d'interférer avec les fonctions des lysosomes [15]. Soyons confiants quant au fait que ces nouvelles connaissances sur l'infection des cellules par le SARS-CoV-2 auront un impact sur la prise en charge thérapeutique des patients atteints de la COVID-19.

This way out! SARS-CoV-2 takes the lysosomal pathway

\section{REMERCIEMENTS}

National Institutes of Health RO1 Ey015625 (Michael S. Marks et Graça Raposo), Fondation pour la recherche médicale (labellisation équipe FRM EQU201903007827, Graça Raposo), Fondation $A R C$ pour la recherche sur le cancer (ARCPJA22020060002267, Cédric Delevoye), ANR «MYOACTIONS» (ANR-17-CE11-0029-03, Anne Houdusse et Cédric Delevoye), GENESPOIR (Cédric Delevoye), LabEx Cell( $n$ )Scale (ANR-11LABX-0038), Inserm, Institut Curie, et CNRS. 


\section{LIENS D'INTÉRÊT}

Les auteurs déclarent n'avoir aucun lien d'intérêt concernant les données publiées dans cet article.

\section{RÉFÉRENCES}

1. Iseni F, Tournier JN. Une course contre la montre: création du SARS-CoV-2 en laboratoire, un mois après son émergence! Med Sci (Paris) 2020 ; $36: 797-802$.

2. Robinson M, Schor S, Barouch-Bentov R, et al. Viral journeys on the intracellular highways. Cell Mol Life Sci $2018 ; 75: 3693-714$.

3. Ghosh S, Dellibovi-Ragheb TA, Kerviel A, et al. $\beta$-coronaviruses use lysosomes for egress instead of the biosynthetic secretory pathway. Cell $2020 ; 183$ 1520-35.el4.

4. Shang J, Ye G, Shi K, et al. Structural basis of receptor recognition by SARS-CoV-2. Nature 2020 ; $581: 221-4$.

5. Hofmann I, Munro S. An N-terminally acetylated Arflike GTPase is localised to lysosomes and affects their motility. / Cell Sci 2006 ; 119 : 1494-503.
6. Hetz C, Zhang K, Kaufman RJ. Mechanisms, regulation and functions of the unfolded protein response. Nat Rev Mol Cell Biol 2020 ; 21 : 421-38.

7. Chu H, Chan CM, Zhang X, et al. Middle East respiratory syndrome coronavirus and bat coronavirus HKU9 both can utilize GRP78 for attachment onto host cells. J Biol Chem 2018 ; 293 : 11709-26.

8. Ha DP, Van Krieken R, Carlos AJ, et al. The stressinducible molecular chaperone GRP78 as potential therapeutic target for coronavirus infection. J Infect $2020 ; 81: 452-82$

9. Delevoye C, Marks MS, Raposo G. Lysosome-related organelles as functional adaptations of the endolysosomal system. Curr Opin Cell Biol 2019 ; 59 : 147-58.

10. Langemeyer L, Fröhlich F, Ungermann C. Rab GTPase function in endosome and lysosome biogenesis. Trends Cell Biol $2018 ; 28$ : 957-70.

11. Agola J0, Hong L, Surviladze Z, et al. A competitive nucleotide binding inhibitor: in vitro characterization of Rab7 GTPase inhibition. ACS Chem Biol 2012 ; 7 : 1095-108.
12. Friedman JR, Dibenedetto JR, West $M$, et al. Endoplasmic reticulum-endosome contact increases as endosomes traffic and mature. Mol Biol Cell 2013 ; $24: 1030-40$.

13. Wang R, Simoneau CR, Kulsuptrakul J, et al. Genetic screens identify host factors for SARS-CoV-2 and common cold coronaviruses. Cell 2021 ; 184 : 106-19. el4.

14. Miserey-Lenkei S, Trajkovic K, D’Ambrosio JM, et al. A comprehensive library of fluorescent constructs of SARS-CoV-2 proteins and their initial characterization in different cell types. Biol Cell 2021 ; doi : 10.1111/ boc. 202000158

15. Miao G, Zhao H, Li Y, et al. ORF3a of the COVID-19 virus SARS-CoV-2 blocks HOPS complex-mediated assembly of the SNARE complex required for autolysosome formation. Dev Cell 2021 ; 56 : 427-42. e5.

\section{NOUVELLE}

\section{Troubles des conduites alimentaires}

\section{Rôle des interneurones cholinergiques du striatum}

Salah El Mestikawy ${ }^{1,2}$, Mathieu Favier ${ }^{1}$

\author{
lDépartement de psychiatrie, \\ Institut universitaire en santé mentale Douglas, \\ Université McGill, 6875 boulevard Lasalle, H4H 1R2 Montréal, \\ Québec, Canada. \\ ${ }^{2}$ Sorbonne université, Université Pierre et Marie Curie UMR 119 \\ - CNRS UMR 8246 - Inserm U1130, Neurosciences Paris Seine - \\ Institut de biologie Paris Seine, Paris, France. \\ mathieu.favier@mail.mcgill.ca
}

$>$ Les troubles des conduites alimentaires tels que l'anorexie mentale, la boulimie ou l'hyperphagie boulimique sont des maladies psychiatriques graves [1]. L'anorexie mentale est difficile à traiter, en particulier chez l'adulte, et présente le taux de mortalité (5 à $10 \%$ ) le plus élevé de tous les troubles mentaux [2]. L'anorexie mentale se caractérise par une restriction persistante de l'apport alimentaire (anorexie mentale de type restrictif) ou par une consommation excessive de nourriture suivie d'épisodes de purge. La boulimie mentale et l'hyperphagie boulimique se définissent par la consommation d'une grande quantité de nourriture sur une courte période de temps, accompagnée d'une perte de contrôle sur la prise alimentaire. Les troubles des conduites alimentaires sont fréquemment associés à un manque de flexibilité cognitive, à une perturba- tion du système de la récompense, ou à un trouble obsessionnel-compulsif. L'incapacité des patients à interrompre le comportement alimentaire anormal en dépit de ses conséquences négatives suggère une base commune avec d'autres troubles compulsifs [3]. Malgré la gravité de ces troubles des conduites alimentaires, il n'existe actuellement aucun traitement biologique spécifique. Chez l'homme ou chez la souris, chaque action reflète un équilibre entre deux types d'apprentissages: les comportements dirigés par un but et les comportements habituels (habitudes) [4]. Les comportements dirigés par un but sont observés lorsque les individus sont engagés dans une action visant à atteindre un objectif spécifique. Dans ce cas, la sélection de l'action est régie par son résultat. Les comportements dirigés par un but se caractérisent par une prise en compte des conséquences futures et une flexibilité adaptative aux changements de l'environnement. La répétition, avec succès, des comportements dirigés par un but entraîne une automatisation de ces comportements et la formation d'habitudes, qui sont donc façonnées par l'expérience passée. Contrairement aux comportements dirigés par un but, les habitudes sont découplées du résultat et sont enclenchées par les stimulus environnementaux. Elles se caractérisent par l'efficacité et la rigidité [4]. L'automaticité des habitudes permet au cerveau de libérer des ressources attentionnelles et décisionnelles, mais elle peut également être préjudiciable, menant à l'apparition de comportements automatiques inadaptés, qui peuvent aboutir à des troubles compulsifs [5]. Nous avons cherché à déterminer si un déséquilibre entre les comportements dirigés par 